\title{
„Durch humor-Symptome (,Feuchtigkeit'-Symptome) gekennzeichnete epidemische Wärme-Erkrankung" (shiwen 濕瘟): Untersuchung der klinischen Merkmale von Covid-19 in Shanghai
}

\author{
Xue Yan, Zhang Wei, Xu Guihua, Chen Xiaorong, Lu Yunfei, Wang Zhenwei, Shi Kehua, Wu Huan,
} Yu Jian

Shanghai Journal of Traditional Chinese Medicine and Pharmacology (Shanghai Zhongyiyao Zazhi), 5. März 2020

\author{
Auszugsweise aus dem Chinesischen ins Englische übersetzt und überarbeitet \\ von Heiner Frühauf \\ Aus dem Englischen übersetzt von Sepp Leeb, München
}

\begin{abstract}
Zusammenfassung: Der Artikel geht auf Covid-19 aus Sicht der klassischen Chinesischen Medizin ein. Aufgrund ihrer klinischen Erfahrung mit der Erkrankung ordnen die Autoren sie der Kategorie "durch humor-Symptome (,Feuchtigkeit'-Symptome) gekennzeichnete epidemische Wärme-Erkrankung" (shiwen 濕瘟) innerhalb der "WärmeErkrankungen“ (wenbing) zu. Sie erläutern die in den Medi-
\end{abstract}

zinklassikern für epidemische Krankheiten verwendete Nomenklatur und beschreiben den Krankheitsmechanismus, bei dem humor („Feuchtigkeit", shi) im Vordergrund steht. Anhand der Symptome in den vier verschiedenen Stadien der Erkrankung erläutern sie das jeweilige pathologische Geschehen.

Schlüsselwörter: Covid-19 · Durch humor-Symptome („Feuchtigkeit“-Symptome) gekennzeichnete epidemische WärmeErkrankung (shiwen) · Coronavirus · Wärme-Erkrankung (wenbing) · Epidemische Wärme-Erkrankungen“ (wenyi) · Humor („Feuchtigkeit", shi) · Calor („Hitze“, re)

Chin Med 2020;35:143-149

\begin{abstract}
“Epidemic febrile diseases characterised by symptoms of dampness (humor)" (shiwen): examination of the clinical features of Covid-19 in Shanghai
\end{abstract}

\begin{abstract}
The article looks at Covid-19 from the perspective of classical Chinese Medicine. On the strength of their clinical experience with the illness, the authors assign it to the category of epidemic febrile diseases characterised by dampness
\end{abstract}

(humor) (shiwen 濕瘟) within the group of "warm diseases" (wenbing). They explain the nomenclature used in the classical medical works for epidemic diseases and describe the mechanisms of the disease in which dampness (humor, shi) plays a prominent role. Describing the symptoms occurring in the four different stages of the disease, they explain the pathogenesis of each stage.

Key Words: Covid-19 · Epidemic febrile diseases characterised by dampness (humor) symptoms (shiwen) · Corona virus · Warm diseases (wenbing) · Epidemic warm fevers (wenyi) · Dampness (humor, shi) · Heat (calor, re) 
Zum Begriff „Wärme-Erkrankung“ in der Chinesischen Medizin

Die klimatischen Bedingungen
In der Nomenklatur der traditionellen Chinesischen Medizin ist die Bezeichnung „Wärme-Erkrankung“ (wenbing 温病) ein Oberbegriff für alle Arten von Erkrankungen, die außen induziert sind und die in ihren Symptommanifestationen die Anzeichen einer calor-Erkrankung („Hitze“-Erkrankung, rebing) aufweisen. Dazu gehören auch calor-Erkrankungen („Hitze“-Erkrankungen, rebing) mit hochansteckenden und epidemischen Eigenschaften. „Epidemische WärmeErkrankungen“ (wenyi 瘟疫1) sind eine Untergruppe von Wärme-Erkrankungen und der spezielle Terminus für calor-Heteropathien („Hitze“-Schrägläufigkeiten, rexie), die leicht übertragbar sind und eine Pandemie auslösen können. Aufgrund unserer klinischen Erfahrungen bei der Behandlung von über 100 bestätigten Covid-19-Fällen in Shanghai sind wir zu der Ansicht gelangt, dass diese Epidemie der traditionellen Kategorie „durch humor-Symptome (,Feuchtigkeit'-Symptome) gekennzeichnete epidemische Wärme-Erkrankung“" (shiwen 濕瘟) zuzuordnen ist.

\section{Zuordnung zu den Wärme-Erkrankungen (wenbing)}

Covid-19 brach gegen Ende des den zyklischen Zeichen ji hai 已亥 zugeordneten Jahres 2019 aus, das als der wärmste Zeitraum innerhalb des gesamten 60-jährigen Zyklus innerhalb des traditionellen kalendarischen Systems der Himmelsstämme und Erdzweige gilt. Im Winter herrscht normalerweise kaltes Wetter, aber der Winter dieses Jahres war warm und sorgte für ungewöhnliche klimatische Bedingungen, die vermutlich den Ausbruch dieser Krankheit begünstigten. Im Kapitel „Oberes Calorium (oberer Wärmebereich, shangjiao)" in der "Systematischen Differenzierung von Wärme-Erkrankungen“ (Wenbing tiaobian, 1798 von Wu Tang, auch Wu Jutong, verfasst) heißt es unmissverständlich: „Der Winter soll kalt sein, doch ist er stattdessen warm, kommt es nicht zum (notwendigen) Untertauchen und zur Speicherung des Yang, infolgedessen wird die Bevölkerung von Wärme-Erkrankungen befallen. “ An anderer Stelle weist der Autor Wu Tang 吴瑭 (1758-1836) darauf hin, dass „Wärme-Erkrankungen in ventus-WärmeErkrankungen (,Wind'-Wärme, fengwen 风温), calor-Wärme-Erkrankungen (,Hitze'-Wärme, wenre 温热) und epidemische Wärme-Erkrankungen (wenyi 温疫) unterteilt werden können ... alle Pathogene, die Wärme-Erkrankungen hervorrufen, dringen durch die Nase und den Mund ein und breiten sich von dort tiefer und weiter nach unten aus." Dementsprechend hat die Regierung in ihrem Standardbehandlungsansatz deutlich gemacht, dass diese Krankheit mittels feiner Tröpfchen erfolgt, die ihren Ursprung in den Atemwegen haben. Überdies brach die Krankheit zuerst im Winter aus und wurde von dem neuartigen Coronavirus verursacht, einem Pathogen mit hochansteckenden und pandemischen Eigenschaften - genau wie in Wu Youkes 吴又可 (1582-1652) klassischer Definition einer Epidemie: „Epidemische Wärme-Erkrankungen (wenyi) können in jeder Jahreszeit auftreten. Die Epidemie ( $y i$ ) zieht das die Seuche auslösende Qi (liqi 瘀气) zwischen Himmel und Erde heran ... und wenn es ankommt, wird jeder davon infiziert, der damit in Berührung kommt - ob er nun alt oder jung, schwach oder kräftig ist."

\footnotetext{
1 Zu beachten ist, dass das gleiche Zeichen für wen 温 "Wärme“ hier mit dem Radikal ",Krankheit" \% statt mit dem Radikal „Wasser" *ै geschrieben wird.
} 


\section{Toxisches (du) und humor („Feuchtigkeit“, shi) als weitere Kennzeichen}

Toxisches ( $d u$ 毒) ist zudem eine Bezeichnung für Heteropathien („Schrägläufigkeiten“, xie) von großer Gefährlichkeit. Covid-19 weist pathogene Merkmale auf, die als humoröse toxische Heteropathie („feucht-toxische“ Schrägläufigkeit, shiduxie) bezeichnet werden können. Der Ming-zeitliche gelehrte Arzt Ye Tianshi 叶天士 (1667-1747) hat einmal gesagt: „In unserer Region von Wu 吴 (entspricht in etwa der heutigen Provinz Jiangsu bei Shanghai) sind humor-Heteropathien (,Feuchtigkeit'-Schrägläufigkeiten, shixie) weit verbreitet.“ Shanghai ist eine Stadt am Meer; die dortige Bevölkerung isst gern nahrhafte, süße und fettige Lebensmittel; sie arbeiten zu viel und ruhen sich zu wenig aus. Daher begünstigt ihr genereller Lebensstil die Ausbildung innerer calor-humidus-Zustände („Feuchtigkeit-Hitze“, shire) oder zumindest eine rasche Herausbildung von humor („Feuchtigkeit“, shi) und stagnierendem calor („Hitz““, re), sobald sich das Virus festgesetzt hat. Zugleich hat es in diesem Winter erheblich mehr geregnet als in den vergangenen Jahren - ein weiterer Faktor, der die Entwicklung von humorHeteropathien („Feuchtigkeit“-Schrägläufigkeiten, shixie) begünstigt.

In der Diagnostik der Schule der morbi temperati (Wärme-Erkrankungen, wenbing) wird mehr Wert auf die Untersuchung der Zunge gelegt als auf die traditionellere Pulstastung. Eine Gruppe von Forschern, die die Zungen bestätigter Covid-19-Patienten untersucht haben, stellten fest, dass die meisten davon eine rote bis dunkelrote Färbung aufwiesen, begleitet von einem Belag, der weiß und klebrig oder gelb und klebrig oder dick und gelb und klebrig war - lauter Anzeichen für calor humidus („Feuchtigkeit-Hitze“, shire) (Lu 2020).

Zudem kann die Inkubationszeit dieses Virus potenziell bis zu 14 Tage betragen, wesentlich länger als bei den meisten anderen epidemischen Krankheiten. Auch dauert nach einer Ansteckung die Periode mit niedrigem Fieber ebenfalls relativ lange. Diese langsam voranschreitenden Eigenschaften sind ein weiterer Hinweis auf eine Ätiologie, an der humor-Heteropathien („Feuchtigkeit“-Schrägläufigkeiten, shixie) mitwirken. Infolge der Beteiligung toxischer epidemischer Heteropathien („Schrägläufigkeiten“, xie) ist für die Krankheit zugleich ein rasches Voranschreiten typisch; zudem entwickelt sich im Spätstadium den o. pulmonalis (Fk „Lunge“, fei) blockierendes calor-Toxisches (redu bifei), und es kommt zu einer Blockade im Inneren und Entweichen nach außen (neibi waituo 内闭外脱) ${ }^{2}$.

\section{Zum Begriff „,durch humor-Symptome gekennzeichnete epidemische Wärme-Erkrankung“" (shiwen 濕瘟)}

In Kapitel 58 des „Klassikers der schwierigen Probleme“ (Nanjing, 1./2. Jh. n. u. Z.) heißt es: „Es gibt fünf verschiedene Arten von (epidemischen) algor-Erkrankungen (shanghan): ,Getroffensein von Wind' (zhongfeng) ${ }^{3}$, algor laedens (,schädigende

\footnotetext{
2 Hierbei handelt es sich um eine kritische Situation, in der aus Sicht der Chinesischen Medizin zu den lebensbedrohlichen inneren Blockaden die gefürchtete "Separation von feinstofflichem mingmen-Qi vom materiellen Körper" hinzukommt, wofür in der „Abhandlung über schädigende Kälte (algor laedens)" (Shanghan lun, von Zhang Zhongjing, aus dem 2. Jh.) die klassische Rezeptur "Das Dekokt gegen die vier Kontravektionen" (Sini tang, s. Porkert 1994, S. 241) und später das „Einzig aus Ginseng hergestellte Dekokt“ (Dushen tang, s. Hempen 2006, S. 330) verschrieben worden sind.

3 "Getroffensein von Wind“ (zhongfeng) steht hier für ein von ventus („Wind”, feng) dominiertes Grundmuster der yang-maior-Schicht (Großes Yang, taiyang) und ist nicht zu verwechseln mit der anderen Verwendung zur Bezeichnung eines ventus internus (innerer "Wind", neifeng) mit apolektischen Erscheinungen.
}

Die Rolle von humor

(„Feuchtigkeit“, shi)

Mit welcher Geschwindigkeit die Erkrankung fortschreitet 
Klassische Textstellen zu epidemischen Krankheiten

Kombination von humor

(,Feuchtigkeit", shi) und calor

(„Hitze“, re)
Kälte', shanghan $)^{4}$, humor-Wärme-Erkrankung (,Feuchtigkeit'-Wärme, shiwen 濕温), calor-Erkrankung (rebing) und Wärme-Erkrankung (wenbing); die Leiden, die sie verursachen, sind alle unterschiedlich.“ Deshalb behandelt der „Klassiker der schwierigen Probleme" (Nanjing) die Kategorien humor-Erkrankung („Feuchtigkeit“, shi), calor-Erkrankung („Hitze“, re) und algor-Erkrankung („Kälte“, han) unter dem Oberbegriff algor laedens („,schädigende Kälte“, shanghan), der hier im weitesten Sinn des Wortes verwendet wird. In Ge Hongs 葛洪 (283-343) „Rezepturen für den Notfall, die unter dem Ellenbogen zu tragen sind“ (Zhouhou beiji fang) aus dem 4. Jahrhundert heißt es im Kapitel 13 mit dem Titel „Rezepturen für die Behandlung von algor laedens (,schädigende Kälte), jahreszeitlich bedingten Epidemien und Wärme-Erkrankungen" (Zhi shanghan shiqi wenbing fang): „Algor laedens (,schädigende Kälte', shanghan), jahreszeitlich bedingte Krankheiten (shixing) und Wärme-Epidemien (wenyi) sind drei verschiedene Bezeichnungen für denselben Krankheitstyp - im Grund ihres Wesens unterscheiden sie sich nur geringfügig." Mehrere Gelehrte haben deshalb auf die Möglichkeit hingewiesen, dass mit der Störung, die im 2. Jahrhundert als humor-Wärme-Erkrankung (shiwen 湿温5) bezeichnet wird, vor allem Epidemien gemeint sind, die von humor-Heteropathien („Feuchtigkeit“-Schrägläufigkeiten, shixie) ausgelöst werden, hochansteckend sind und mit hoher Sterblichkeit einhergehen (Zhang 2015).

In einem Werk aus dem 19. Jahrhundert, „Klassische Theorien und moderne Interpretationen zum Thema Wärme-Erkrankungen" (Wenre jingwei), heißt es in dem Kapitel mit dem Titel „Dr. Xue Shengbais Ansatz bei humor-WärmeErkrankungen (,Feuchtigkeit'-Wärme-Erkrankungen)" (Xue Shenghai shire bing pian 薛生白湿热病篇) ganz ähnlich: „Typisch für das Syndrom einer humorWärme-Erkrankung (,Feuchtigkeit'-Wärme, shiwen) ist die Anwesenheit epidemischer ansteckender (Heteropathien) (yili 疫瘀) im Inneren.“ Zhong Shaotaos 钟少桃 1931 erschienenes Lehrbuch „Lehrmaterialien für das Studium der Theorien der Schule der Wärme-Erkrankungen“ (Wenbingxue jiangyi) fügt dem hinzu: „Man kann sagen, dass eine humor-Wärme-Erkrankung (,Feuchtigkeit'-Wärme, shiwen) ... diagnostiziert werden kann, solange (das Vorhandensein von) calor (,Hitze', re) in humor (,Feuchtigkeit', shi) oder (von) humor (,Feuchtigkeit', shi) in calor (,Hitze', re) festgestellt werden kann - unabhängig davon, zu welcher Jahreszeit sie auftritt.“ Alle oben aufgeführten Textstellen deuten darauf hin, dass für eine humor-Wärme-Erkrankung („Feuchtigkeit“-WärmeErkrankung, shiwen) Symptome typisch sind, die humor („Feuchtigkeit“, shi) mit calor („Hitze“, re) verbinden.

\section{Unterschied zwischen „Wärme-Erkrankung“ (wen 溫) und „epidemischer Wärme-Erkrankung“ (wen 瘟)}

Sowohl in der Vergangenheit als auch heute hat es zahlreiche Debatten über den Unterschied zwischen den Termini „epidemische Wärme-Erkrankung (wen 瘟)“ und „Wärme-Erkrankung (wen 溫)“ gegeben. Im Wörterbuch „Eine Sammlung von Reimen“ (Jiyun) aus dem 11. Jahrhundert heißt es, dass das Zeichen wen 瘟

\footnotetext{
4 Algor laedens („schädigende Kälte", shanghan) steht hier für algor laedens ("schädigende Kälte“, shanghan) im engeren Sinne, für ein von algor („Kälte", han) dominiertes Grundmuster der yangmaior-Schicht (Großes Yang, taiyang).

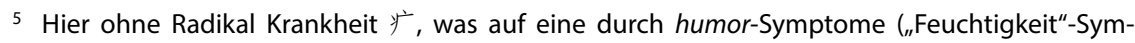
ptome) gekennzeichnete Entität des Wenbing hindeutet.
} 
ausdrücklich für epidemische Erkrankungen steht. ,瘟 wird wie 溫 ausgesprochen und steht für epidemische Krankheiten." Um nun wieder auf das Coronavirus zurückzukommen, das hochansteckend und gefährlich ist und pandemisches Potenzial hat, ist der Begriff 瘟 unserer Ansicht nach am besten dafür geeignet, die oben beschriebenen Eigenschaften von Covid-19 zu bezeichnen. Daher schlagen wir vor, diese spezielle Epidemie als einen Typus einer „durch humor-Symptome gekennzeichneten epidemische Wärme-Erkrankung“( shiwen 濕瘟) zubezeichnen.

\section{$5 \quad$ Lokalisation und Krankheitsmechanismus}

Der ursprüngliche Ort der Erkrankung ist der o. pulmonalis (Funktionskreis „Lunge“, fei), von dem sie sich zum „Membran-Ursprung“ (moyuan 膜原) ${ }^{6}$ und zum o. tricalorii (Drei Wärmebereiche, sanjiao) ausbreiten kann. Grundsätzlich lässt sich die Krankheit deshalb als eine komplexe Mischung von humor („Feuchtigkeit“, shi), Toxisches $(d u)$, calor („Hitze“, re) und Stagnation $(y u)$ beschreiben. Zuerst sind die typischsten Symptome Fieber, Husten und Keuchatmung. In Kapitel 37 des „Angelpunktes der Struktivkraft“ im „Inneren Klassiker des Gelben Fürsten“ (Huangdi Neijing Lingshu, etwa 1. Jh. v. u. Z., später überarbeitet) heißt es: „Eine Erkrankung des o. pulmonalis (Fk ,Lunge', fei) zeigt sich in Form von Keuchatmung und geblähten Nasenflügeln." Weiter steht im 47. Kapitel des „Angelpunktes der Struktivkraft“ (Lingshu): „Ist das qi pulmonale (Qi des Fk,Lunge', feiqi) zu weit oben, dann ist es kontravektiv nach oben gestiegen, was sich in schulterschüttelndem Husten manifestiert."

Wenn die Krankheit weiter voranschreitet, kann sich humor-Toxisches („Feuchtigkeit“-Toxisches“, shidu) einstauen und zu schwelendem calor („Hitze“, re) entwickeln und auf diese Weise eine Situation schaffen, in der sich humor („Feuchtigkeit“, shi), calor („Hitze“, re) und Toxisches (du) verbinden und in den „Membran-Ursprung“ (moyuan 膜原) eindringen. Diese Entwicklung führt in der Regel zu Erstickungsgefühlen in der Brust, unangenehmem Geschmack im Mund, Übelkeit und Erbrechen usw. Das ist genau der Prozess, der vor Jahrhunderten in der „Abhandlung über epidemische Wärme-Erkrankungen“ (Wenyi lun, von Wu Youxing 1642 verfasst) beschrieben wurde: „Die Heteropathie (Schrägläufigkeit, xie) dringt durch Nase und Mund ein und nistet sich ein, indem sie ,den Membran-Ursprung umschließt', wie es das Lingshu nennt." Deshalb verursacht das Eindringen dieses Typs von humor-toxisch epidemischer Heteropathie („feucht"-toxische epidemische Schrägläufigkeit, shidu yixie) die innere Entwicklung von stagnierendem calor („Hitze“, re) im Inneren, der das obere Calorium (oberer Wärmebereich, shangjiao) befallen und sich dann auf das mittlere Calorium (mittlerer Wärmebereich, zhongjiao) und sogar auf das untere Calorium (unterer Wärmebereich, xiajiao) ausweiten kann und auf diese Weise die typischen Symptome hervorruft, die da sind: hohes Fieber, Atemstörungen, trockener Mund, Beklemmungsgefühle in der Brust, Unruhe, Harnverhalt, Durchfallneigung und andere Anzeichen der systematischen Ausbreitung einer Heteropathie (Schrägläufigkeit, xie) in alle drei Calorien (Wärmebereiche, jiao).

6 Der Begriff „Membran-Ursprung” (moyuan 膜原) kann zwei Bedeutungen haben: Nach dem Kap. 39 des Suwen bezeichnet er den Bereich zwischen Pleura und Diaphragma. In der Schule der "Wärme"-Erkrankungen wird er als Bereich zwischen extima (Oberfläche, biao) und intima (Inneres, li) gesehen und gilt auch als „Pforte zum o. tricalorii (Drei Wärmebereiche, sanjiao)", Anm. des Übers. s. Liu 2001:186.
Die ersten Symptome

Ausweitung auf alle drei Calorien (Wärmebereiche, jiao) 
Kombination mehrerer ätiologischer Einflüsse

Schädigung von Qi und Yin

\section{$6 \quad$ Verschiedene Stadien einer Covid-19-Erkrankung}

Das ätiologische Voranschreiten von Covid-19 erfolgt üblicherweise folgendermaßen: Im Anfangsstadium der Infektion weisen die Patienten Symptome von Schüttelfrost auf, die sich oft zu Fieber mit trockenem Husten weiterentwickeln, begleitet von einer Zungenfärbung, die entweder blassrot, rot oder dunkelrot ist, und einem Belag, der entweder gelb und klebrig ist oder weiß und klebrig, mit der Tendenz, gelb und klebrig zu werden. Diese Anzeichen deuten alle darauf hin, dass sich humor-Toxisches in den o. pulmonalis („Feuchtigkeit“-Toxisches in den Fk „Lunge“, shidu yufei) einstaut. Anschließend weisen die meisten Patienten Trockenheit von Mund und Rachen auf, eine rote oder dunkelrote Zungenspitze sowie einen Zungenbelag, der einerseits klebrig ist, zugleich wegen Mangels an Säften aber auch rissig. Alle diese Anzeichen deuten auf eine Entwicklung hin, bei der sich das humor-Toxische ("Feuchtigkeit“- Toxisches, shidu) bereits in calor („Hitze“, re) und ariditas („Trockenheit“, zao) umgewandelt haben. Das zweite Krankheitsstadium ist deshalb dadurch gekennzeichnet, dass noch humorToxisches („Feuchtigkeit“-Toxisches, shidu) besteht und eingestauter humor („Feuchtigkeit“, shi) jetzt aber zugleich auch calor („Hitze“, re) hervorruft. An diesem kritischen Punkt verbinden sich die ätiologischen Einflüsse von humor („Feuchtigkeit“, shi), calor („Hitze“, re) und Toxisches $(d u)$ und breiten sich im ganzen Körper aus, indem sie in den „Membran-Ursprung“ (moyuan) und die drei Calorien (Wärmebereiche, jiao) eindringen.

Im dritten und gravierendsten Stadium dringen humor („Feuchtigkeit“, shi), Toxisches $(d u)$ und calor („Hitze“, re) in die Schicht des qi constructivum (Bauenergie, ying) ein, wo sie stark bewegen und eine depletio xue (energetische Schwäche des Xue) hervorrufen (haoxue dongxue). An diesem Punkt verbinden sich diese drei auch noch mit dem Element der Xue-Stase $(y u)$, wodurch die Erkrankung in das lebensbedrohliche Stadium einer Blockade im Inneren und Entweichen nach außen (neibi waituo 内闭外脱, s.o.) eintreten kann.

In der Genesungsphase weisen die Patienten meistens Symptome auf, die auf eine duale depletio (energetische Schwäche, $x u$ ) von Qi und Yin (qi yin liangxu) hindeuten. Im Kapitel „Schadensbehebung“ (Sunfu) der „Abhandlung über epidemische Wärme-Erkrankungen“ (Wenyi lun, s.o.) weist Wu Youke schon vor vierhundert Jahren darauf hin, dass „diese Heteropathien (Schrägläufigkeiten, xie) zuallererst und vor allem das Qi schädigen." Es gilt als unbestritten, dass die humoröse und toxische Natur dieser Krankheit die Gesamtenergie eines Menschen deutlich schädigt. Darüber hinaus beeinträchtigt der toxische calor („Hitze“, re) den o. pulmonalis (Fk „Lunge“, fei), indem er sein Qi und Yin schädigt. Dazu kommt, dass der lange und massive Einsatz von Arzneimitteln, die humor („Feuchtigkeit“, shi) ausleiten, die Säfte schädigen und das Yin dezimieren können. Die typische, allgemein übliche Zuführung von intravenösen Flüssigkeiten wird das yang lienale (Yang des Fk „Milz“, piyang) schädigen. Das sind die Hauptgründe, weshalb die meisten Patienten in der Genesungsphase Anzeichen einer depletio qi et yin pulmonale et lienale (stomachi) (energetische Schwäche von Qi und Yin der Fk „Lunge“ und „Milz“ (und „Magen“), fei pi (wei) qi yin xu) aufweisen.

\section{Literatur}

Hempen C-H, Fischer S, Hummelsberger J, Koch A, Leonhardy H, Nögel R, Thede C, Wullinger M: Leitfaden Chinesische Rezepturen. München: Elsevier, 2006.

Liu Guohui. Warm Diseases. A Clinical Guide: Eastland Press, 2001. 
Lu Yunfei, Yang Zongguo, Wang Mei et al. „Wushili xinxing guanzhuang bingdu ganran de feiyan huanzhe zhongyi linchuang tezheng fenxi" (An Analysis of the Clinical Characteristics of Fifty COVID-19 Cases from a Chinese Medicine Perspective), Shanghai Zhongyiyao Daxue Xuebao (18. Februar 2020).

Porkert M: Klassische chinesische Rezeptur. Dinkelscherben: Phainon, 1994.

Zhang Meng, Wang Xiaomei, Liu Qing et al. „Shiwen gainian bianxi“ (A Differential Analysis of the Concept of Damp Febrile Disease), in Zhongyiyao xuebao 45/3 (2015), S. 1-4.

\title{
Zum Autor
}

Heiner Frühauf ist Founding Professor des College of Classical Chinese Medicine an der National University of Natural Medicine in Portland, Oregon. Er studierte Sinologie in Tübingen, Shanghai, Hamburg, Chicago und Tokyo. Im klinischen Bereich ist er Spezialist für die Behandlung von chronischen Krankheiten mit chinesischer Phytotherapie. Er ist Gründer der Weiterbildungsorganisation für klassische chinesische Medizin ClassicalChineseMedicine.org und der phytopharmakologischen Firma Classical Pearls.

\author{
Korrespondenzadresse \\ Prof. Heiner Frühauf \\ College of Classical Chinese Medicine \\ National University of Natural Medicine \\ 049 SW Porter Street \\ Portland, OR 97201, USA
}

\section{Einhaltung ethischer Richtlinien}

Interessenkonflikt: Heiner Frühauf gibt an, dass kein Interessenkonflikt besteht.

Dieser Beitrag beinhaltet keine Studien an Menschen oder Tieren. 


\section{TCM-Quiz 92 \\ von Rainer Nögel}

Frage 1: Yang maior (Großes Yang, taiyang)

a) ist eine metaphorische Qualifikation für die Wandlungsphase Feuer

b) ist die energetische Qualifikation für die c. cardialis (Leitbahn des Fk „Herz“, xin jing)

c) ist die Bezeichnung der äußersten Schicht in der „Abhandlung über schädigende Kälte (algor laedens)“ (Shanghan lun, von Zhang Zhongjing, aus dem 2. Jh.)

d) ist die Bezeichnung für den Extrapunkt Ex2/EX-HN-5 („Die Sonne“)

e) ist eine Bezeichnung für Cinnamomi cassiae ramulus (Guizhi)

Frage 2: Anhaltendes Grübeln führt zu
a) einer Belastung der oo. lienalis et stomachi (Fk „Milz“ und „Magen“, pi wei)
b) einer Überwältigung des o. hepaticus (Fk „Leber“, gan)
c) einer Schwächung des o. pulmonalis (Fk „Lunge“, fei)
d) einer Schwächung des 0 . cardialis $(\mathrm{Fk}, \mathrm{Herz}$ “, xin)
e) einer Bezwingung des 0 . renalis (Fk „Niere“, shen)

Frage 3: Dem qi ascitum (Qi der erworbenen Konstitution, houtian zhi qi) zugeordnet bzw. entsprechend ist das
a) qi defensivum (Wehrenergie, weiqi)
b) qi constructivum (Bauenergie, yingqi)
c) qi originale (Ursprungs-Qi, yuanqi)
d) qi genuinum (genuines Qi, zongqi)
e) qi cardiale (Qi des Fk „Herz“, xinqi)

Frage 4: Das qi originale (Ursprungs-Qi, yuanqi) kann am besten beeinflusst werden bei
a) IC4/Di4 („Vereinte Täler“, hegu)
b) P9/Lu9 („Großer Wasserschlund“, taiyuan)
c) L4/Mi4 („Enkel des Herzogs“, gongsun)
d) F40/Gb40 („Das Feld am Hügel“, qiuxu)
e) V65/Bl65 („Der geschnürte Knochen“, shugu)

Frage 5: Houttuyniae herba cum radice (Yuxingcao)
a) hat einen fischähnlichen Geruch
b) ist ein m. refrigerantium desinfectantium (kühlendes und Toxisches herauslösendes Arzneimit- tel, qingre jiedu yao)
c) hat durch seinen scharfen Sapor (Geschmacksrichtung, wei) eine emporhebende Wirkrichtung
d) ist eine nützliche Ergänzung für das „Phragmites-Dekokt“ (Weijing tang)
e) ist Bestandteil des „Pulvers mit Lonicera und Forsythia“ (Yinqiao san)

Frage 6: Die mm. transformatoria pituitae et tussostatica („Schleim“ umwandelnde, Husten stillende und Keuchen besänftigende Arzneimittel, huatan zhike pingchuan yao) werden zur Wirkungsverstärkung
a) häufig durch Rösten mit Honig (mizhi) aufbereitet
b) häufig durch Rösten unter Wenden (chao) aufbereitet (Samen)
c) durch kurzes Kochen (chan) aufbereitet (Armeniacae semen, Xingren)
d) durch Brennen (duan) aufbereitet (mineralische Arzneimittel)

Frage 7: Bei calor venti o. pulmonalis („Wind-Hitze“ im Fk „Lunge“, fei fengre) empfiehlt sich
a) Ingwer
b) Drachenbrunnentee mit Chrysanthemenblüten
c) Pfefferminztee
d) Rettichsaft
e) Miso

Auflösung der Fragen auf Seite 162 